\title{
Heuristic Approach to the Critical Dynamics of the Ising Model
}

\author{
P.R. Silva and V. B. Kokshenev \\ Departamento de Física, ICEx, Universidade Federal de Minas Gerais, \\ Caixa Postal 702, CEP 30123-970, Belo Horizonte, Minas Gerais, Brazil
}

Received on 1 November, 2000

\begin{abstract}
We discuss order-disorder phase transitions of classical and quantum Ising models on the basis of the Ginzburg-Landau-Wilson free-energy action and a modification of a scaling method proposed by Thompson. The two-time critical relaxation scenario, driven by thermal and quantum fluctuations, respectively, is given in terms of the slow-down critical exponent of the order-parameter excitations.
\end{abstract}

The classical Ising model (IM) and the quantum transverse Ising model (TIM) are generically used to study cooperative phenomena. The three-dimensional $(d=3)$ TIM has several applications to real systems[1], including the description of structural order-disorder phase transitions observed in ferroelectrics at a critical temperature $T_{c}$. In the $d=1$ case, the TIM with nearest-neighbor interactions exhibits $T=0$ longrange-order for small enough transverse fields $\Gamma<\Gamma_{c}$. In the current work we propose a new approach to the problem of the critical dynamics. It is based on a heuristic method introduced by Thompson [2] and developed by one of the authors[3]. Thompson's method is based on the idea that the action, as well as a relevant free energy of the system of many scales of length, are limited functions. This heuristic method was formulated through a set of assumptions (see prescriptions A,B,C in Ref.[2]) that avoid dealing with the renormalization-group (RG) equations of motion.

The critical dynamics of a given system is a manifestation of its dynamical instability and can be treated in terms of the collective Goldstone-type soft mode vanishing at the transition temperature $T_{c}$. More specifically, order-disorder transitions display a two-time relaxation scenario given by relaxation times $\tau_{\alpha}$ with $\alpha=c$ (central mode) and $\alpha=s$ (soft mode) for, respectively, diffusive-type relaxation and oscillatory-type behavior. These are related to the finite-time local orderparameter excitations $\omega_{\alpha}$ of width $\Gamma_{\alpha}=\operatorname{Im} \omega_{\alpha}$. We discuss the critical dynamics in terms of the slowing-down dynamical critical exponents $\Delta$ defined as $\Gamma_{\alpha} \propto\left|\varepsilon_{T}\right|^{\Delta_{\alpha}}$ with $\varepsilon_{T}=\left(T-T_{c}\right) / T_{c}$ as $\varepsilon_{T}$ goes to zero. We take
$\Delta=\nu z$, where $\nu$ stands for the correlation length exponent and $z$ is the growth-law exponent that distinguishes diffusion mechanisms.

If we assume a Brownian mechanism, the $d$ dimensional classical IM predicts diffusive-type relaxation dynamics. Its slowing-down exponent is[3]

$$
\Delta_{c}^{(\text {class })}=z_{c} \nu=\frac{(d+2)}{2(d-1)} \quad \text { for } d \leq 4 \text { and } z=2 .
$$

For the real $d=3$ case, $\Delta_{c}=\frac{5}{4}$ was independently deduced (see Eq.15 in Ref.[4]) for the quantum TIM by the Green function method. This corresponds to for the universal critical behavior of the classical and quantum Ising models[5] for $d>1$.

Besides the critical relaxation dynamics given by the central mode, we discuss the critical oscillatory behavior. In the continuum representation, the action induced by the time-dependent order parameter and associated with the free energy can be given by

$$
\begin{gathered}
A_{T}=\int_{L^{d}}\left(\frac{1}{2}|\nabla \Psi(x)|^{2}+\frac{\varepsilon_{T} r(L)}{2}|\Psi(x)|^{2}\right. \\
\left.+\frac{u(L)}{4}|\Psi(x)|^{4}+\frac{M}{2}\left|\frac{\partial \Psi(x)}{\partial t}\right|^{2}\right) d^{d} x,
\end{gathered}
$$

where $\Psi$ stands for the scalar field conjugate to the order parameter and the integration is performed over a $d$-dimensional hypercube of volume $L^{d}$. The coefficients $r(L)$ and $u(L)$ are not critical. The last term, depending on the effective mass $M$, extends the Ginzburg-Landau-Wilson free-energy action to include oscillatory-type kinetic effects. 
Based on the ideas of Thompson[2], we assume that each term in Eq.(2) is of a finite magnitude. To estimate the critical exponents it is not necessary to evaluate with accuracy the terms of the sum. Therefore, we can simplify our work assuming that any term is of the order of unity. Adopting this scheme and considering a stationary motion of the system, $\Psi=\Psi_{0} e^{i \omega t}$, we have the following estimates for the first and the fourth terms in Eq.(2),

$$
\int|\nabla \Psi(x)|^{2} d^{d} x \sim\left|\int\left(-M \omega^{2} \Psi^{2}(x)\right) d^{d} x\right| \sim 1 .
$$

This can be rewritten as $\left\langle|\Psi|^{2}\right\rangle L^{d-2} \approx$ $M\left\langle|\omega|^{2}\right\rangle\left\langle|\Psi|^{2}\right\rangle L^{d}$, where the angle brackets mean the integration (2). In order to perform the mass scaling, a characteristic length associated with the quantum particle behavior is treated as its Compton wavelength, namely, $\lambda_{c}=\hbar / M c$. This yields $M \propto L^{-1}$ and, hence, $\left\langle|\omega|^{2}\right\rangle^{1 / 2} \propto L^{-1 / 2}$. By introducing the soft-mode frequency $\omega_{s} \propto-\varepsilon_{T}^{\gamma / 2}$ and its width $\Gamma_{s} \propto-\varepsilon^{\Delta_{s}}$ through the relationship $\langle\omega\rangle=\omega_{s}+i \Gamma_{s}$, as well as the correlation length $\xi \propto-\varepsilon^{-\nu}$ near the critical temperature, we have $\omega_{s}^{2}+\Gamma_{s}^{2} \propto-\varepsilon_{T}^{\nu}$. Using the known Fisher scaling equation $(\gamma=\nu(2-\eta))$, and the inequality $\gamma>\nu / 2$, we naturally arrive at the overdamped soft-mode regime, i.e. $\Gamma_{s}>\omega_{s}$, that gives $\Delta_{s}=\nu / 2$. In combination with Eq.(1) for the correlation-length critical exponent $\nu$, this provides the slowing-down critical exponent for the overdamped oscillatory motion of the Ising-like system[5],

$$
\Delta_{s}^{(\text {class })}=\frac{\nu}{2}=\frac{(d+2)}{8(d-1)}, \quad \text { for } \quad d \leq 4 .
$$

One can see that, unlike the case of the relaxation motion regime given in Eq.(1), the oscillatory motion is characterized by non-Brownian superdiffusion dynamics that implies $z=1 / 2$. In real $d=3$ space, it follows from Eq.(4) that $\Delta_{s}=5 / 16=0.313$. It should be pointed out that this is close to $\Delta_{s}^{\prime}=\frac{1}{4}$ associated with the soft-mode instability of the high-symmetry, disordered paraelectric phase deduced from the quantum TIM by the Green function method (see Eq.15 in Ref.[4]). The $T_{c}$-universality of the classical IM and the quantum TIM models, also known for the case of classical and quantum Heisenberg spin models, is due to the fact that thermal fluctuations drive the system through the phase transition. As a consequence, the relaxation mechanism does not depend on the details introduced by quantum, Glauber or Kawasaki dynamics (for dis- cussions, see Ref.[5] ). Meanwhile, this is restricted to dimensions $d>1$.

In the $d=1$ case the TIM with nearest-neighbor interactions exhibits $T=0$ long-range-order near the critical field $\Gamma_{c}$. The transition is driven by quantum fluctuations and can be mapped into the critical behavior of the IM with the dimension increased by one $[6,7,8]$. By studying the $T=0$ fixed-point scaling properties, Continentino demonstrated[10] that the TIM exhibits a dimensional shift from $d$ to $d+z$ with respect to the IM, where $z$ is the growth-law exponent $[9,10]$.

One of the substantial characteristics of the action introduced in Ref.[3] is its Galilean invariance. The effectivity of the classical action (2) to find the critical behavior driven by thermal fluctuations has been demonstrated above. Generally, there is a length $L_{\min }$ corresponding to the lower wavelength (ultraviolet) cutoff. For the classical case (2) one can show that $L_{\min }$ is large enough to avoid a relativistic treatment. On the contrary, the quantum behavior requires the low wavelength regime that can be introduced through the Lorentz invariant action (see Eq.(5) below). We then write the following quantum action endowed with a Lorentzian invariance for the scalar order parameter,

$$
\begin{gathered}
A_{\Gamma}=\int d^{d+1} x\left(\frac{1}{2}\left(\partial_{\mu} \Psi(x)\right)\left(\partial^{\mu} \Psi(x)\right)-\frac{\varepsilon_{\Gamma} r(L)}{2} \Psi^{2}(x)\right. \\
\left.+\frac{u(L)}{4} \Psi^{4}(x)\right),
\end{gathered}
$$

with

$$
\partial_{\mu} \Psi \partial^{\mu} \Psi=\partial_{0} \Psi \partial_{0} \Psi-\partial_{1} \Psi \partial_{1} \Psi-\partial_{2} \Psi \partial_{2} \Psi-\partial_{3} \Psi \partial_{3} \Psi
$$

where $\Psi(x)$ stands for the scalar field, and the integration is performed over some $(d+1)$-dimensional spacetime hypercube of volume $L^{d+1}$. The joint integration in time-like $(\mu=0)$ and space-like $(\mu=1,2,3)$ coordinates is performed in accordance with the relativistic treatment of the problem. As before, $r(L)$ and $u(L)$ are not critical, but $\varepsilon_{\Gamma}=\left|\Gamma_{c}-\Gamma\right| / \Gamma_{c}$ measures a deviation from the critical field.

To reveal the critical behavior in action (5), we also adopt the Thompson method[2]: (A) the absolute value of the each integrated term in Eq.(5) is of order of unity; (B) in the limit $L \rightarrow \infty$, the functions $r(L)$ and $u(L)$ remain finite, and $(\mathrm{C})$ the fluctuation part of the action (5) is of order $\xi_{L}^{-(d+1)}$, where $\xi_{L}^{-1}(=\sqrt{r(L)})$ is the characteristic correlation length.

Within the spirit of the Landau approach to secondorder phase-transitions, we introduce the "equilibrium" order parameter, 


$$
\Psi_{\Gamma}^{2}=\varepsilon_{\Gamma} \Psi_{L}^{2}, \quad \text { for } \Gamma<\Gamma_{c}, \quad \text { and } 0 \text { otherwise, }
$$

with

$$
\Psi_{L}^{2}=r(L) / u(L) \text { and } \xi=\left(\varepsilon_{\Gamma} r(L)\right)^{-1 / 2} \equiv\left(\varepsilon_{\Gamma}\right)^{-1 / 2} \xi_{L}
$$

Within the Landau scheme, $r(L)$ and $u(L)$ are constants and the critical exponents associated with the order parameter, $\beta$, and the correlation length, $\nu$, are equal, i.e., $\beta=\nu=\frac{1}{2}$. According to the renormalization-group recipe, the critical fluctuations are accounted through the correlation length. Thus, Eq.(6) provides the self-consistent equation $\xi=$ $\left(\varepsilon_{\Gamma} r(\xi)\right)^{-1 / 2}$ for the diverging correlation length $\xi \sim$ $\varepsilon_{\Gamma}^{-\nu_{\Gamma}}$. Application of the prescription A to the first term in Eq.(5) provides

$$
\int_{L^{d+1}} \nabla \Psi(x)^{2} d^{d+1} x \sim L^{(d+1)-2}<\Psi^{2}>\sim 1
$$

and, thus, $\left\langle\Psi^{2}>\sim L^{2-(d+1)}\right.$. For the second term, one has

$$
\int_{L^{d+1}} \varepsilon_{\Gamma} r(L) \Psi^{2}(x) d^{d+1} x \sim \varepsilon_{\Gamma} r(L) L^{d+1}<\Psi^{2}>\sim 1,
$$

that gives $\varepsilon_{\Gamma} r(L) L^{2} \sim 1$. Finally, the third term yields

$$
\int_{L^{d+1}} u(L) \Psi^{4}(x) d^{d+1} x \sim u(L) L^{d+1}<\Psi^{2}>^{2} \sim 1,
$$

where we have adopted a weak-correlation assumption, $<\Psi^{4}>\approx<\Psi^{2}>^{2}$. By applying the result $(7),\left\langle\Psi^{2}>\right.$ $\sim L^{2-(d+1)}$, to Eq.(9), in combination with the prescription $B$, we have

$u(L) \sim L^{(d+1)-4}$, for $d+1<4$, and $\sim 1$, if $d+1 \geq 4$.

We see that there is a special dimension, given by $d+1=4$, above which the exponents become classical. From relation $(7),\left\langle\Psi^{2}\right\rangle \sim L^{2-(d+1)}$, it follows that the dimension, given by $d+1=2$, is also special. In both cases, we expect logarithmic corrections. Note that, by putting $L=\xi$ in relation (8), we obtain the $\mathrm{RG}$ result (6).

To use the prescription $\mathrm{C}$ we introduce the normalized fluctuation order parameter, $\varphi^{2}=\Psi^{2} / \Psi_{L}^{2}$. Thus, the quantum action (5) is given by

$$
A_{\Gamma}=\frac{r(L)}{2 u(L)} a_{\Gamma},
$$

with

$$
a_{\Gamma}=\int_{L^{d+1}}\left((\nabla \varphi)^{2}-\varepsilon_{\Gamma} r(L) \varphi^{2}+\frac{r(L)}{2} \varphi^{4}\right) d^{d+1} x .
$$

We treat $a_{\Gamma}$ as the fluctuation part of the quantum action. Application of the prescription $\mathrm{C}$ means that $a_{\Gamma}$ $\sim \xi_{L}^{-(d+1)}=[r(L)]^{\frac{d+1}{2}}$. Substituting into Eq.(11), and using Eq.(10) along with the relation $A_{\Gamma} \sim 1$ (due to the prescription $\mathrm{A}$ ), we have

$r(L) \sim L^{\frac{2(d+1)-8}{(d+1)+2}}$, for $d+1 \leq 4$, and, $\sim 1$, if $d+1 \geq 4$.

Solving Eq.(6) for $\xi$, and taking into account that $\xi \sim \varepsilon_{\Gamma}^{-\nu_{\Gamma}}$, we arrive at our main result,

$$
\nu=\frac{(d+1)+2}{4[(d+1)-1]},
$$

for $d+1 \leq 4$, and

$$
\nu=\frac{1}{2}, \quad \text { if } \quad d+1 \geq 4 .
$$

This result can also be written in terms of the slowdown critical exponent,

$$
\Delta_{c}^{(\text {quant })}=z \nu=\frac{d^{*}+2}{4\left(d^{*}-1\right)}
$$

for $d^{*} \leq 4$ with

$$
z=1 \quad \text { and } \quad d^{*}=d+z .
$$

Otherwise $\left(d^{*} \geq 4\right)$, we come back to the classical result $\nu=\frac{1}{2}$ and $z=1$. From Eqs.(1) and (14), one can see that the quantum-TIM critical behavior can be mapped into the classical IM critical behavior by the formal transformation $d \rightarrow d+z$. This is consistent with the dimensional shift predicted by Continentino[10].

To conclude, we have discussed order-disorder phase transitions within the framework of classical and quantum Ising models on the basis of the Ginzburg-LandauWilson action within the heuristic scaling method proposed by Thompson. The critical behavior of the classical Ising model near the non-zero critical temperature (for dimensions $d>1$ ) and of the quantum Ising model $(d \geq 1)$ near the critical transverse field, which are driven by thermal and quantum fluctuations, respectively, are described in terms of the correlation-length $\nu$ and the growth-law $z$ critical exponents. The two-time order-parameter relaxation scenario is given in terms of the slow-down $\Delta=\nu z$ critical exponent of the orderparameter excitations.

This work was supported by the Brazilian agency CNPq. 


\section{References}

[1] R.V. Stinchcombe, J. Phys. C6, 2459 (1973).

[2] C.J. Thompson, J. Phys. A9, L25 (1976).

[3] P.R. Silva, Phys., Stat. Sol. (b) 166, K103 (1991).

[4] V.B. Kokshenev, M.C. Nemes and J.I. Kim, Solid State Commun. 98421 (1996).
[5] V.B. Kokshenev and P.R. Silva, Mod. Phys. Lett. B 12, 265 (1998).

[6] P. Pfeuty, Ann. Phys. 57, 79 (1970).

[7] A.P. Young, J. Phys. C8, L309 (1975).

[8] M. Suzuki, Prog. Theor. Phys. 56, 1454 (1976).

[9] J.A. Hertz, Phys. Rev. B14, 1165 (1976)

[10] M. A. Continentino, Phys. Rep. 239, 181 (1994). 